\title{
Steady State Analysis of Balanced-Allocation Routing
}

\author{
Aris Anagnostopoulos* Ioannis Kontoyiannis ${ }^{\dagger}$ \\ Eli Upfal*
}

November 20, 2018

\begin{abstract}
We compare the long-term, steady-state performance of a variant of the standard Dynamic Alternative Routing (DAR) technique commonly used in telephone and ATM networks, to the performance of a path-selection algorithm based on the "balanced-allocation" principle 13, 17); we refer to this new algorithm as the Balanced Dynamic Alternative Routing (BDAR) algorithm. While DAR checks alternative routes sequentially until available bandwidth is found, the BDAR algorithm compares and chooses the best among a small number of alternatives.

We show that, at the expense of a minor increase in routing overhead, the BDAR algorithm gives a substantial improvement in network performance, in terms both of network congestion and of bandwidth requirement.
\end{abstract}

\footnotetext{
${ }^{*}$ Computer Science Department, Brown University, Box 1910, Providence, RI 029121910, USA. E-mail: \{aris, eli\}@cs.brown. edu. Supported in part by NSF grants CCR0121154, and DMI-0121495.

${ }^{\dagger}$ Division of Applied Mathematics and Department of Computer Science, Brown University, Box F, 182 George St., Providence, RI 02912, USA. E-mail: yannis@cs. brown.edu Web: www.dam.brown.edu/people/yannis/. Supported in part by NSF grant \#0073378CCR and USDA-IFAFS grant \#00-52100-9615.
} 


\section{Introduction}

Fast, high bandwidth, circuit switching telecommunications systems such as ATM and telephone networks often employ a limited path-selection algorithm in order to fully utilize the network resources while minimizing routing overhead. Typically, between each pair of nodes in the network there is a dedicated bandwidth for communication, namely, no more than a certain fixed number of calls can be simultaneously active between each pair of nodes. This dedicated bandwidth is chosen in order to satisfy the demand for communication between these stations. Only when this bandwidth is exhausted the admission control protocol tries to find an alternative route through intermediate nodes. To minimize overhead and routing delays, the protocol checks just a small number of alternative routes; if there are no free connections available on any of these alternatives, then the call or communication request is rejected. Implementations that use this technique include the Dynamic Alternate Routing (DAR) algorithm used by British Telecom [7], and AT\&T's Dynamic Nonhierarchical Routing (DNHR) algorithm [1].

A common feature in these (and other) currently implemented protocols is the sequential examination of alternative routes. Only when the algorithm examines a route and finds it cannot be used an alternative one is examined. The criteria for when a route can or should be used, and the method in which the alternative route is selected have been the subject of extensive research, in particular, in the context of British Telecom's DAR algorithm [6, 7, 8]; see Kelly [9] for an extensive survey.

Dynamic routing can be viewed as a special case of the on-line load balancing problem, where the load (incoming calls or requests) may be assigned to one or more servers (network links), and jobs (communication requests) can be scheduled only on specific subsets (paths) of the set of servers, as defined by the network topology. In this paper we study the impact of replacing the sequential searches of the routing algorithm by a version of the balanced allocation principle. The basic idea is as follows: Instead of sequentially choosing alternative options (in our case, paths) until a desirable one is found, in the balanced-allocation regime the algorithm randomly chooses and examines a number of possible options, and assigns the job at hand to the option which appears to be the best at the time of the assignment.

A number of papers have demonstrated the advantage of the application of the balanced allocation-principle [2, 3, 4, 17, 18] for standard load balancing problems, where jobs require only one server and can be executed by any server in the system. This research has shown that balanced allocations 
usually produce a very substantial improvement in performance, at the cost of a small increase in overhead: Since several alternatives are examined even when the first alternative would have been satisfactory, the complexity of the routing algorithm is increased. But, as has been shown before and as we also demonstrate in the present context, examining even a very small number of alternative (thus increasing overhead by a very small amount) can offer great performance improvements.

The idea of employing the balanced allocation principle to the problem of dynamic network routing as described in this paper was first explored in [12]. In this context the goal is to reduce system congestion and minimize the blocking probability, that is, the probability that a call request is rejected. The main difficulty in applying and analyzing the balanced allocation principle in a network setting is in handling the dependencies imposed by the topology of the network. The preliminary results in 12 show that the advantage of balanced allocations is so significant that it holds even in the presence of a set of dependencies.

The performance of a routing protocol can be analyzed in a static (finite, discrete time) or in a dynamic (infinite, continuous time) setting. The static case has been extensively studied in [11], extending and strengthening the results in [12]. In this paper we consider the continuous-time case. The analysis of the continuous-time case suggested in [12] was based on applying Kurtz's density-dependent jump Markov chain technique, following the supermarket model analysis in [17, 18]. However, since the argument there is incomplete [10], we present here a different analysis. Our results concern the long-term behavior of large networks employing a routing protocol based on the balanced allocations principle. The main tools we employ are a Lyapunov drift criterion used to establish the existence of a stationary distribution for the BDAR routing protocol, and a continuous-time extension of the technique in [3], used to analyze the stationary behavior of a network.

Balanced allocations have also been studied in the context of queueing networks, where analogous results (under different asymptotic regimes than the ones in this paper) are obtained in [17, 22, 13, 21], among others.

\subsection{Model Description and Main Results}

In the types of networks considered in this paper, a logical link or "bandwidth" is reserved between each pair of stations, and an alternative route is only used when this logical link has already been exhausted. We model such a network as the complete graph $G=(V, E)$ with $|V|=n$ vertices (stations) and $|E|=N=\left(\begin{array}{l}n \\ 2\end{array}\right)$ edges (links). 
The input to the system is a sequence of call requests, which are assumed to arrive at Poisson times: New calls onto each link (i.e., between each pair of nodes) arrive according to a Poisson process with rate $\lambda$, all arrival streams being independent. Similarly, the duration of a call is independent of all arrival times all other call durations, and it is exponentially distributed with mean $1 / \mu$.

The routing algorithm has to process the calls on-line, that is, the $t$-th request is either assigned a path or rejected before the algorithm receives the $(t+1)$-th request. Once a call is assigned to a path, that path cannot be changed throughout the duration of the call. We assume that each edge has a capacity of $2 B$ calls, where half of this capacity is reserved for direct links (namely it will only be used for call requests between these two nodes), and the other half is reserved for being used as part of an alternative route between two stations.

As in most of our results we consider large networks with a number $n$ of nodes growing to infinity, we will also assume that the capacity parameter $B$ may vary with $n$. Specifically, we assume that $B=B_{n}$ is nondecreasing in $n$, and we also allow the possibility $B=\infty$.

The goal in designing an efficient routing protocol is to assign routes to the maximum possible number of call requests without violating the capacity constraints on the edges. We will compare the performance of the following two protocols:

The d-Dynamic Alternative Routing (DAR) algorithm works as follows. When a new call request arrives, it tries to route the call through the direct (one-link) path. If there is no available bandwidth on the direct path, then the algorithm sequentially chooses alternative routes of length two and assigns the call to the first available path. Up to $d$ such choices are made, and they are made at random. If no possible path is found, then the request is rejected.

The d-Balanced Dynamic Alternative Routing (BDAR) algorithm also assigns a new call request to the direct path if there is available bandwidth. If not, then the algorithm chooses $d$ length-two alternative paths at random, and compares the maximum load among them (where the load of such a path is taken to be the maximum load of the two links on that path). Then the call is assigned to the path with the minimum load. As before, if there is no path with free bandwidth among these $d$ choices, then the call is rejected.

The model described so far, together with one of the two protocols above, induces a continuous-time stochastic process describing the behavior of the network. As we show below, this system (for fixed $n$ ) converges to a sta- 
tionary regime exponentially fast. For our purposes, the main performance measure is the minimum required bandwidth that ensures that, under the stationary distribution of the network, the blocking probability (i.e., the probability that a new call is rejected) is appropriately small.

In this paper our main goal is to compare the performance of the DAR algorithm with that of BDAR. It is clear that BDAR's performance is dominated by its performance on alternative (length-two) routes. Therefore, in order to simplify the analysis, we consider a variant of BDAR, called $\mathrm{BDAR}^{*}$, which ignores the direct links and services each call only via an alternative route, making use only of the $B$ alternative connections of each edge. In other words, we assume that each edge has capacity $B$ and all of it is dedicated to alternative routes. We show that even though the BDAR* policy ignores the direct links, it has superior performance compared to DAR.

The following result illustrates this superiority by exhibiting explicit asymptotic bounds on their bandwidth requirements. It follows from the results in Theorems 0 and 6 .

Theorem 1. Assume that all the edges have a capacity of $2 B$ links.

Under the DAR policy, edge capacity

$$
B=\Omega\left(\sqrt{\frac{\ln n}{d \ln \ln n}}\right), \quad \text { as } n \rightarrow \infty
$$

is necessary to ensure that a new call is not lost with high probability.

On the other hand if we perform the BDAR* policy (thus ignoring the $B$ direct links), edge capacity

$$
B=\frac{\ln \ln n}{\ln d}+o\left(\frac{\ln \ln n}{\ln d}\right), \quad \text { as } n \rightarrow \infty
$$

suffices to ensure that a new call is not lost with high probability.

In the above result and throughout the paper, we say that a limiting statement holds "with high probability" (abbreviated "whp.") if it holds with probability that is at least $1-1 / n^{c}$ for some constant $c>0$. For example, when we say that a random variable " $X_{n}=O(\ln n)$ whp." we mean that there are positive constants $C$ and $c$ such that $\operatorname{Pr}\left(X_{n} \leq C \ln n\right) \geq$ $1-1 / n^{c}$ for all $n$ large enough. Similarly, $X_{n}=o(\ln n)$ whp. means that there is a $c>0$ such that, for all $\epsilon>0, \operatorname{Pr}\left(X_{n} \leq \epsilon \ln n\right) \geq 1-1 / n^{c}$ for all $n$ large enough.

Note that the result of Theorem 1 is exactly analogous to that obtained in [11] in the discrete-time case. 


\section{Analysis of Balanced-Allocation Routing}

This section presents the main contribution of this paper, a steady state analysis of the performance of the BDAR* routing algorithm. The network is a complete graph with $n$ nodes and $N=\left(\begin{array}{l}n \\ 2\end{array}\right)$ undirected edges. New calls arrive at Poisson times with rate $\lambda$ and their durations are exponentially distributed with mean $1 / \mu$, as described earlier. As it turns out, an important parameter in the analysis of the network load is the ratio $\rho=\lambda / \mu$.

\subsection{Unbounded capacities}

We first analyze the maximum load on edges when the algorithm is used on a network with unbounded edge capacity, corresponding to $B=B_{n}=\infty$. This model induces a continuous time Markov process $\boldsymbol{\Phi}=\{\Phi(t): t \geq$ $0\}$, where $\Phi(t)=\left(l_{1}(t), l_{2}(t), \ldots, l_{N}(t)\right)$, and each $l_{i}(t)$ denotes the load, at time $t$, of the $i$ th link in the network. As we show next, this Markov process has a stationary distribution $\pi_{n}$ to which it converges exponentially fast, regardless of the initial state of the network. We then prove a high probability bound on the maximum load on any edge in the system under this stationary distribution.

Since we are only interested in the load of the alternative paths on the edges, each state of this Markov process corresponds to the load on edges from a collection of length-two paths. We say that a vector $x=$ $\left(l_{1}, l_{2}, \ldots, l_{N}\right)$ is a legal state if it corresponds to the load on the $N$ edges from a collection (possibly empty) of length-two paths. The natural state space $\Sigma$ for our process $\boldsymbol{\Phi}$ is then taken to be

$$
\Sigma=\left\{x=\left(l_{1}, l_{2}, \ldots, l_{N}\right) \mid l_{i} \in \mathbb{N}, \quad x \text { is a legal state }\right\} .
$$

The process $\boldsymbol{\Phi}$ evolves on $\Sigma$ according to the model described above. This evolution is formalized by the transition semigroup $\left\{P^{t}: t \geq 0\right\}$ of $\boldsymbol{\Phi}$, where $P^{t}(x, y)$ is simply the probability that $\boldsymbol{\Phi}$ is in state $y$ at time $t$ given that it was in state $x$ at time zero, $P^{t}(x, y)=\operatorname{Pr}\{\Phi(t)=y \mid \Phi(0)=x\}$.

Our first result shows that $\boldsymbol{\Phi}$ has a stationary (or invariant) distribution to which it converges exponentially fast. It is stated in terms of the "Lyapunov function" $V(x)$ which is defined as $1+$ (total number of active calls in state $x)$ :

$$
V(x)=V\left(l_{1}, l_{2}, \ldots, l_{N}\right)=1+\frac{1}{2} \sum_{i=1}^{N} l_{i} .
$$


Theorem 2. Assume that the BDAR* algorithm is used on a network with $n$ nodes, each of which has infinite capacity. Then the induced Markov process $\Phi$ has an invariant distribution $\pi_{n}$, and, moreover, for any initial state $x \in \Sigma$, the distribution of $\Phi(t)$ converges to $\pi_{n}$ exponentially fast, namely there is a constant $\gamma<1$, such that

$$
\sup _{y}\left|P^{t}(x, y)-\pi_{n}(y)\right| \leq V(x) \gamma^{t}, \text { for all } t \geq 0 \text { and all } x \in \Sigma .
$$

Proof. Our proof uses the Lyapunov drift criterion for the exponential ergodicity of a continuous time Markov processes [14, 5, 15]. To state our main tool we recall a few definitions, adapted to our case of countable state space.

The generator $\mathcal{A}$ of the process $\boldsymbol{\Phi}$ is a linear operator on functions $F$ : $\Sigma \rightarrow \mathbb{R}$ defined by

$$
\mathcal{A} F(x)=\lim _{h \downarrow 0} \frac{\mathrm{E}\left(F(\Phi(h)) \mid x_{0}=x\right)-F(x)}{h}
$$

whenever the above limit exists for all $x \in \Sigma$. The explosion time of $\boldsymbol{\Phi}$ is defined as

$$
\zeta=\sup _{n} J_{n}
$$

where

$$
J_{0}=0, \quad J_{n+1}=\inf \left\{t \geq J_{n}: \Phi_{t} \neq \Phi_{J_{n}}\right\}
$$

$\left(J_{0}, J_{1}, \ldots\right.$ are the jump times of the Markov process). We say $\boldsymbol{\Phi}$ is nonexplosive if $\operatorname{Pr}\left(\zeta=\infty \mid \Phi_{0}=x\right)=1$ for any starting state $x$.

The following theorem follows from the more general results in [15, 5], specialized to the case of a continuous-time Markov process with a countable state space.

Theorem 3. 15, 5] Suppose a Markov process evolving on a countable state space that is nonexplosive, irreducible (with respect to the counting measure on $\Sigma$ ) and aperiodic. If there exists a finite set $C \subset \Sigma$, constants $b<\infty$, $\beta>0$ and a function $V: \Sigma \rightarrow[1, \infty)$, such that,

$$
\mathcal{A} V(x) \leq-\beta V(x)+b \mathbb{I}_{C}(x) \quad x \in \Sigma,
$$

then the process is positive recurrent with some invariant probability measure $\pi$, and there exist constants $\gamma<1, D<\infty$ such that

$$
\sup _{y}\left|P^{t}(x, y)-\pi(y)\right| \leq D V(x) \gamma^{t}, \text { for all } t \geq 0 \text { and all } x \in \Sigma .
$$


It is easy to verify that the process is $\psi$-irreducible and aperiodic, with the maximal aperiodicity measure $\psi$ being the counting measure on $\Sigma$.1 Also the process is nonexplosive since the number of new calls in a given interval has a Poisson distribution with a finite mean, therefore the probability of infinite number of transition in a finite interval is 0 .

To show that the drift criterion (2) can satisfied, we use the Lyapunov function $V(x)=1+($ total number of active calls in state $x)$ defined in (1) above.

In order to compute $\mathcal{A} V$ we notice that when a new call enters the system, it increases the loads of two edges by 1 , hence the value of $V$ by 1 , and when a call terminates the value of $V$ decreases by 1 . Therefore, new calls are generated with rate $\lambda N$ and calls are terminated at a rate $\mu(V(x)-1)$. The probability that in a time interval $h$ there are 2 or more new calls or terminations of calls is $o(h)$. 2 Using these observations we can compute $\mathcal{A} V$ :

$$
\begin{aligned}
\mathcal{A} V(x) & =\lim _{h \downarrow 0} \frac{V(x)+\lambda N \cdot h-\mu \cdot(V(x)-1) \cdot h+o(h)-V(x)}{h} \\
& =\lambda N-\mu V(x)+\mu
\end{aligned}
$$

To analyze the drift condition we distinguish between the following two cases:

- $x \in C$ :

$$
\mathcal{A} V(x)=\lambda N-\mu V(x)+\mu<-\frac{\mu V(x)}{2}+\lambda N+\mu
$$

- $x \in C^{c}(x$ is in the complement of $C)$ :

$$
\mathcal{A} V(x)=\lambda N-\mu V(x)+\mu \leq \frac{\mu V(x)}{2}-\mu V(x)=-\frac{\mu V(x)}{2} .
$$

Thus, the drift condition holds for $\beta=\mu / 2$ and $b=\lambda N+\mu$.

Having shown the existence of an invariant limiting distribution $\pi_{n}$, we now analyze the maximum load on the edges under this distribution.

\footnotetext{
${ }^{1}$ This follows along the lines of the arguments in Chapters 4 and 5 of 16. In particular, note that all sets $\{y\} \in \Sigma$ are $\nu_{1}$-small and $P^{1}(x, y)>0$ for all $x, y \in \Sigma$ so that in fact $\boldsymbol{\Phi}$ is irreducible and strongly aperiodic.

${ }^{2}$ Here and in the next expression with the notation $o(h)$ we mean that $f$ is $o(h)$ if $\lim _{h \rightarrow 0} \frac{f(h)}{h}=0$. In the rest of the text $o(n)$ has the usual meaning.
} 
Theorem 4. Consider a network with $n$ nodes, and let $\pi_{n}$ be the invariant distribution of the induced Markov process under the BDAR* policy with unbounded edge capacity. Under $\pi_{n}$, the maximum number of calls in any edge is bounded whp. by

$$
\frac{\ln \ln n}{\ln d}+o\left(\frac{\ln \ln n}{\ln d}\right), \quad \text { as } n \rightarrow \infty .
$$

Proof. In order to compute the maximum edge load under the stationary distribution, we start observing the system at some time point and study its transient behavior; we then use the results to deduce the properties of the invariant distribution. In particular, we show that there exists a constant $T=O\left(n \frac{\ln \ln n}{\ln d}\right)$, such that for any state of the system at time $\tau-T$ that has sufficiently large probability, whp. at time $\tau$ the maximum number of calls on any edge is

$$
\frac{\ln \ln n}{\ln d}+o\left(\frac{\ln \ln n}{\ln d}\right)
$$

The high level idea is the following: We partition the time $T$ into $\frac{\ln \ln n}{\ln d}+$ $o\left(\frac{\ln \ln n}{\ln d}\right)$ periods of length $O(n)$. Roughly, we argue that at the end of the $i$-th period, whp., for each node, the number of incident edges with load greater than $i$ is at most $\alpha_{i}$. The $\alpha_{i}$ decrease doubly exponentially, so at the end of the last period we will be able to deduce that there are no edges with load more than $\frac{\ln \ln n}{\ln d}$ whp. The challenge is to handle the dependencies, as the number of calls during some period depends on the number of calls of the previous periods. We now proceed with the details.

Suppose that a call routed at time $t$ is assigned to edges $e_{1}$ and $e_{2}$. The height of that call at edge $e_{1}$ is 1 plus $l_{e_{1}}(t-)$. We define the following random variables:

- $L_{\geq i}^{v}(t)$ : Number of edges incident to node $v$ with load at least $i$ at time $t$.

- $M_{\geq i}^{v}(t)$ : Number of calls at edges incident to $v$ with height greater or equal to $i$ at time $t$.

Trivially we have $L_{\geq i}^{v}(t) \leq M_{\geq i}^{v}(t)$.

We define the sequence of values $\left\{a_{i}\right\}$ which decreases doubly exponen- 
tially:

$$
\begin{aligned}
& \alpha_{\kappa}=\frac{(n-1) \rho}{\kappa} \\
& \text { where } \kappa=e \cdot \sqrt[d-1]{2 \rho \cdot 4^{d}} \\
& \alpha_{i}=\frac{2 \rho \cdot 4^{d} \cdot a_{i-1}^{d}}{(n-1)^{d-1}} \\
& \text { for } i>0 \text { and } \alpha_{i-1} \geq \sqrt[d]{\frac{1}{\rho} n^{d-1} \ln n}, \\
& \alpha_{i^{*}}=25 \ln n \\
& i^{*} \text { is the smallest } i \text { for which } a_{i-1}<\sqrt[d]{\frac{1}{\rho} n^{d-1} \ln n} \\
& \alpha_{i^{*}+1}=10
\end{aligned}
$$

Solving the recurrence we get for $\kappa \leq i<i^{*}$,

$$
\begin{aligned}
\alpha_{i+\kappa} & =\frac{\left(2 \rho \cdot 4^{d}\right)^{\frac{d^{i}-1}{d-1}}}{\kappa^{d^{i}}}(n-1)=\frac{1}{\sqrt[d-1]{2 \rho \cdot 4^{d}}} \cdot\left[\frac{\sqrt[d-1]{2 \rho \cdot 4^{d}}}{\kappa}\right]^{d^{i}}(n-1) \\
& =\frac{1}{\sqrt[d-1]{2 \rho \cdot 4^{d}}} \cdot \frac{n-1}{e^{d^{i}}}
\end{aligned}
$$

and for the $i^{*}$

$$
\alpha_{i^{*}-1}<\sqrt[d]{\frac{1}{\rho} n^{d-1} \ln n}
$$

which gives

$$
i^{*}=\frac{\ln \ln n}{\ln d}+o\left(\frac{\ln \ln n}{\ln d}\right) .
$$

Next we define $T=n\left(i^{*}+2\right)=O\left(n \frac{\ln \ln n}{\ln d}\right)$ and an increasing sequence of points in time: let $t_{\kappa}=\tau-T$ and for $i>\kappa, t_{i}=t_{i-1}+n$, so that the end of the last period, $t_{i^{*}+2}$, is the current time $\tau$.

Let $E$ denote the event "at time $t_{\kappa}$ there are at most $(1+\epsilon) N \rho$ calls in the system," and let

$$
C_{i}=\left\{\forall v \in V, t \in\left[t_{i}, T\right]: M_{\geq i}^{v}(t) \leq 2 a_{i}\right\} .
$$

We will show by induction that for $i=\kappa, \ldots, i^{*}+1$

$$
\operatorname{Pr}\left(\neg C_{i} \mid E\right) \leq \frac{2 i}{n^{2}}
$$

For the base case $(i=\kappa)$, conditioning on $E$, the expected number of calls for a particular node $v$ is $(1+\epsilon)(n-1) \rho$, since each existing call has probability $2 / n$ to have $v$ as an endpoint. Hence, by using the Chernoff bound

$$
\operatorname{Pr}(\text { node } v \text { has more than }(1+\delta)(n-1) \rho \text { calls } \mid E)=o\left(1 / n^{c}\right)
$$


where $\epsilon<\delta<1$ and $c$ can be any positive constant. Therefore

$$
\operatorname{Pr}\left(\neg C_{\kappa} \mid E\right) \leq n \operatorname{Pr}\left(M_{\geq \kappa}^{v}>\frac{2(n-1) \rho}{\kappa} \mid E\right)<\frac{2 \kappa}{n^{2}} .
$$

For the induction step we assume that

$$
\operatorname{Pr}\left(\neg C_{i-1} \mid E\right) \leq \frac{2(i-1)}{n^{2}}
$$

Let $G$ denote the event "a new call is generated with $v$ as an endpoint," and call $u$ the other endpoint and $w$ the intermediate node of the alternative path. We have

$\operatorname{Pr}$ (a new call increases $\left.M_{\geq i}^{v} \mid G, C_{i-1}, E\right)$

$\leq \operatorname{Pr}$ (height of new call is $\geq i$ in either $(v, w)$ or $\left.(w, u) \mid G, C_{i-1}, E\right)$

$\leq\left(\frac{L_{\geq i-1}^{v}+L_{\geq i-1}^{u}}{n-1}\right)^{d}$

$\leq\left(\frac{M_{\geq i-1}^{v}+M_{\geq i-1}^{u}}{n-1}\right)^{d} \quad$ since $L_{\geq i}^{v}(t) \leq M_{\geq i}^{v}(t)$

$\leq\left(\frac{2 \cdot 2 \alpha_{i-1}}{n-1}\right)^{d} \triangleq q_{i} \quad$ from the induction hypothesis

Notice that for $i=\kappa, \ldots, i^{*}$ we have

$$
q_{i} \leq \frac{\alpha_{i}}{2 \rho(n-1)}
$$

We now define

$$
F_{i}=\left\{\forall v \in V: M_{\geq i}^{v}\left(t_{i}\right)<\alpha_{i}\right\}
$$

and prove Lemmas 1 and 3 , that allow us to conclude that $\operatorname{Pr}\left(\neg C_{i} \mid E\right) \leq \frac{2 i}{n^{2}}$.

Lemma 1. Under the inductive hypothesis

$$
\operatorname{Pr}\left(\neg F_{i} \mid C_{i-1}, E\right) \leq \frac{1}{n^{2}}
$$


Proof. Consider the time interval $\left[t_{i-1}, t_{i}\right]$ and recall that $t_{i}-t_{i-1}=n$.

First notice that since the duration of each call follows an exponential distribution with parameter $\mu$, the probability that a call that is already in the system at time $t_{i-1}$ will remain until the end of the interval $t_{i}$ is $e^{-n \mu}$. Hence all these calls will end before the end of the interval with exponentially high probability. To analyze the number of the remaining calls that were created during the period we make use of lemma 2 which completes the proof of the lemma.

Lemma 2. Consider a period of length $\Delta$ and a given node $v$. Conditioning on $C_{i-1}$, the number of new calls that increased $M_{\geq_{i}}^{v}$ when they were generated, and remained until the end of the period is less than $\alpha_{i}$, with probability at least $1-\frac{1}{n^{4}}$.

Proof. Each node has $n-1$ incident links in each of which new calls are generated with rate $\lambda$. Conditioning on having a new request on $v, M_{>i}^{v}$ is increased with probability at most $q_{i}$. Therefore the number of calls at time $t_{i}$ is stochastically dominated by that formed by a process that generates new calls with rate $\lambda(n-1) q_{i}$ which have a duration exponentially distributed with parameter $\mu$. This process is the same as the infinite server Poisson queue [19, page 18] in which the number of calls at the end of the period is distributed according to a Poisson distribution with rate

$$
\lambda(n-1) q_{i} \Delta p
$$

where

$$
p=\int_{0}^{\Delta} \frac{e^{-\mu(\Delta-x)}}{\Delta} \mathrm{d} x=\frac{1}{\mu \Delta}\left(1-e^{-\mu \Delta}\right) \leq \frac{1}{\mu \Delta}
$$

So the rate is at most $\lambda q_{i}(n-1) / \mu=\rho q_{i}(n-1)$.

We now distinguish the following two cases:

Case 1: For $i \leq i^{*}$, by using Equation 5 we get that the expected number of calls at the end of the period is at most $\alpha_{i} / 2$ and by applying a Chernoff bound for the Poisson distribution, we get that the probability that the number of calls is higher than $a_{i}$ is bounded by

$$
\frac{e^{-\frac{\alpha_{i}}{2}}\left(e \frac{\alpha_{i}}{2}\right)^{\alpha_{i}}}{\alpha_{i}^{\alpha_{i}}}=e^{-\left(\ln 2-\frac{1}{2}\right) \alpha_{i}}
$$

\footnotetext{
${ }^{3}$ see for example [20, page 416].
} 
For $i<i^{*}$ we have from the definition of $\alpha_{i}$

$$
e^{-\left(\ln 2-\frac{1}{2}\right) \alpha_{i}}=e^{-\left(\ln 2-\frac{1}{2}\right) \frac{2 \rho \cdot 4^{d} \alpha_{i-1}^{d}}{(n-1)^{d-1}}}=e^{-\left(\ln 2-\frac{1}{2}\right) \frac{2 \rho \cdot 4^{d} \frac{1}{\rho} n^{d-1} \ln n}{(n-1)^{d-1}}}=o\left(\frac{1}{n^{4}}\right),
$$

while for $i=i^{*}$ we get

$$
e^{-\left(\ln 2-\frac{1}{2}\right) \alpha_{i}}=e^{-\left(\ln 2-\frac{1}{2}\right) 25 \ln n}=o\left(\frac{1}{n^{4}}\right) .
$$

Case 2: For $i=i^{*}+1$, using Equation 1 we get that the expected number of calls at the end of the period is at most

$$
\frac{4^{d} \cdot \alpha_{i-1}^{d}}{(n-1)^{d}} \rho(n-1)=\frac{(4 \cdot 25 \ln n)^{d}}{(n-1)^{d-1}} \rho
$$

and we get the high probability result with the Chernoff bound.

Lemma 3. Under the inductive hypothesis

$$
\operatorname{Pr}\left(\neg C_{i} \mid C_{i-1}, F_{i}, E\right) \leq \frac{1}{n^{2}}
$$

Proof. We have:

$$
\begin{aligned}
& \operatorname{Pr}\left(\neg C_{i} \mid F_{i}, C_{i-1}, E\right)=\frac{\operatorname{Pr}\left(\neg C_{i} \wedge F_{i} \mid C_{i-1}, E\right)}{\operatorname{Pr}\left(F_{i} \mid C_{i-1}, E\right)} \\
& \leq \frac{n}{\operatorname{Pr}\left(F_{i} \mid C_{i-1}, E\right)} \operatorname{Pr}\left(\exists v \in V, t_{a}, t_{b} \in\left[t_{i}, T\right]:\right. \\
& \leq \frac{\left.M_{\geq i}^{v}\left(t_{a}\right)=\alpha_{i}, M_{\geq i}^{v}\left(t_{b}\right)=2 \alpha_{i}, M_{\geq i}^{v}(t) \geq \alpha_{i} \forall t \in\left[t_{a}, t_{b}\right] \mid C_{i-1}, E\right)}{\operatorname{Pr}\left(F_{i} \mid C_{i-1}, E\right)} \int_{t_{a}=t_{i}}^{T} \int_{t_{b}=t_{a}}^{T} \operatorname{Pr}\left(M_{\geq i}^{v}\left(t_{a}\right)=\alpha_{i},\right. \\
& \leq \frac{\left.M_{\geq i}^{v}\left(t_{b}\right)=2 \alpha_{i}, M_{\geq i}^{v}(t) \geq \alpha_{i} \forall t \in\left[t_{a}, t_{b}\right] \mid C_{i-1}, E\right) \mathrm{d} t_{b} \mathrm{~d} t_{a}}{\operatorname{Pr}\left(F_{i} \mid C_{i-1}, E\right)} \int_{t_{a}=t_{i}}^{T} \int_{t_{b}=t_{a}}^{T} \operatorname{Pr}\left(M_{\geq i}^{v}\left(t_{a}\right)=\alpha_{i},\right. \\
&\left.M_{\geq i}^{v}\left(t_{b}\right)=2 \alpha_{i} \mid M_{\geq i}^{v}(t) \geq \alpha_{i} \forall t \in\left[t_{a}, t_{b}\right], C_{i-1}, E\right) \mathrm{d} t_{b} \mathrm{~d} t_{a}
\end{aligned}
$$

The probability inside the integrals is the probability that the new calls generated during the interval $\left[t_{a}, t_{b}\right]$, increased $M_{\geq i}^{v}$, and remained until the 
end of the interval, are at least $\alpha_{i}$. By applying Lemma 2, we get that this probability is at most $n^{-4}$. Hence

$$
\begin{aligned}
\operatorname{Pr}\left(\neg C_{i} \mid F_{i}, C_{i-1}, E\right) & \leq \frac{n}{1-\frac{1}{n^{2}}} \int_{t_{a}=t_{i}}^{T} \int_{t_{b}=t_{a}}^{T} \frac{1}{n^{4}} \mathrm{~d} t_{b} \mathrm{~d} t_{a} \\
& \leq \frac{n}{1-\frac{1}{n^{2}}} \cdot T^{2} \cdot \frac{1}{n^{4}} \\
& =o\left(\frac{1}{n^{2}}\right)
\end{aligned}
$$

since $T=O\left(n \frac{\ln \ln n}{\ln d}\right)$.

Having proven the two lemmas we can now show that $\operatorname{Pr}\left(\neg C_{i} \mid E\right) \leq$ $2 i / n^{2}$ :

$$
\begin{aligned}
\operatorname{Pr}\left(\neg C_{i} \mid E\right) & =\operatorname{Pr}\left(\neg C_{i} \mid C_{i-1}, E\right) \cdot \operatorname{Pr}\left(C_{i-1}, E\right) \\
& +\operatorname{Pr}\left(\neg C_{i} \mid \neg C_{i-1}, E\right) \cdot \operatorname{Pr}\left(\neg C_{i-1}, E\right) \\
& \leq \operatorname{Pr}\left(\neg C_{i} \mid C_{i-1}, E\right)+\frac{2(i-1)}{n^{2}} \\
& =\operatorname{Pr}\left(\neg C_{i} \mid C_{i-1}, F_{i}, E\right) \cdot \operatorname{Pr}\left(F_{i} \mid C_{i-1}, E\right) \\
& +\operatorname{Pr}\left(\neg C_{i} \mid C_{i-1}, \neg F_{i}, E\right) \cdot \operatorname{Pr}\left(\neg F_{i} \mid C_{i-1}, E\right)+\frac{2(i-1)}{n^{2}} \\
& \leq \frac{1}{n^{2}}+\frac{1}{n^{2}}+\frac{2(i-1)}{n^{2}} \\
& =\frac{2 i}{n^{2}}
\end{aligned}
$$

We have therefore shown that the event $C_{i^{*}+1}$ holds whp. until the end of $T$, which means that for every node $v$, after the $\left(i^{*}+1\right)$-th period, there will be no more than $\alpha_{i^{*}+1}=10$ incident edges with load more than $i^{*}+1$. We will now bound the probability that in the next interval $\left(\left[t_{i^{*}+2}, t_{i^{*}+3}\right]\right.$, the last interval of $T$ ) there will be an incident edge of $v$ with load more than $i^{*}+3$, conditioning on the event $C_{i^{*}+1}$. For this to happen, we must have at least 2 new calls to be routed using one of the 10 high-loaded edges. The probability that two specific new calls use these edges is at most

$$
\left(\frac{10}{n-2}\right)^{2 d}=O\left(\frac{1}{n^{4}}\right),
$$

since $d \geq 2$. The expected number of calls with $v$ as an endpoint is $\lambda(n-1) n$, since $(n-1)$ links are connected to $v$ in each of which new calls are generated 
with rate $\lambda$, while the total length of the interval is $n$. This implies that whp. there will be $O\left(n^{2}\right)$ new calls in the whole period. Combining this fact with Equation 6 and summing for all the nodes we conclude that at the end of period $T$ there will be no edges with load more than $i^{*}+3$ whp.

We now consider the stationary distribution $\pi_{n}$, and show that under it

$$
\operatorname{Pr}\left[l_{\max } \leq \frac{\ln \ln n}{\ln d}+o\left(\frac{\ln \ln n}{\ln d}\right)\right]=1-o(1) .
$$

where $l_{\text {max }}$ denotes the maximum number of calls on any edge. Let $s(t)$ be the state of the system at time $\tau$, and consider the following partitioning of the state space of the underlying Markov process:

- $S_{1}$ : States in which the total number of calls in the system is at most $(1+\epsilon) N \rho$, and the maximum load is at most $\frac{\ln \ln n}{\ln d}+o\left(\frac{\ln \ln n}{\ln d}\right)$.

- $S_{2}$ : States in which the total number of calls in the system is at most $(1+\epsilon) N \rho$, and the maximum load is at least $\frac{\ln \ln n}{\ln d}+\Omega\left(\frac{\ln \ln n}{\ln d}\right)$.

- $S_{3}$ : States in which the total number of calls in the system is more than $(1+\epsilon) N \rho$.

We have shown that

$$
\operatorname{Pr}\left(s(\tau) \in S_{2} \mid s(\tau-T) \in S_{1} \cup S_{2}\right)=o(1)
$$

and we can easily show that

$$
\operatorname{Pr}\left(s(\tau) \in S_{3} \mid s(\tau-T) \in S_{1} \cup S_{2}\right)=o(1)
$$

Moreover in the stationary distribution the number of calls in the system has a Poisson distribution with parameter $N$. Hence by using the Chernoff bound

$$
\sum_{i \in S_{3}} \pi_{i}=o(1)
$$

Then we have

$$
\sum_{i \in S_{2} \cup S_{3}} \pi_{i}=\sum_{i \in S_{2}} \pi_{i}+\sum_{i \in S_{3}} \pi_{i}
$$


The second term is $o(1)$, while for the first one

$$
\begin{aligned}
\sum_{i \in S_{2}} \pi_{i}= & \sum_{j} \operatorname{Pr}\left(s(\tau) \in S_{2} \mid S(\tau-T)=j\right) \cdot \pi_{j} \\
= & \sum_{j \in S_{1} \cup S_{2}} \operatorname{Pr}\left(s(\tau) \in S_{2} \mid S(\tau-T)=j\right) \cdot \pi_{j} \\
& +\sum_{j \in S_{3}} \operatorname{Pr}\left(s(\tau) \in S_{2} \mid S(\tau-T)=j\right) \cdot \pi_{j} \\
= & \sum_{j \in S_{1} \cup S_{2}} \pi_{j} \cdot o(1)+o(1)=o(1)
\end{aligned}
$$

Therefore

$$
\sum_{i \in S_{2} \cup S_{3}} \pi_{i}=o(1)
$$

which implies that

$$
\sum_{i \in S_{1}} \pi_{i}=1-o(1)
$$

and completes the proof of the theorem.

\subsection{Bounded Capacities}

In this section we use the analysis of the BDAR* algorithm for unbounded capacities to compute the bandwidth requirement $B(<\infty)$ that ensures that a new call is not lost whp.

Theorem 5. Assume that all the edges have capacity $B$ circuits which can be a function of $n$. Then if we perform the BDAR* policy, edge capacity

$$
B=\frac{\ln \ln n}{\ln d}+o\left(\frac{\ln \ln n}{\ln d}\right), \quad \text { as } n \rightarrow \infty
$$

ensures that a new call is not lost whp.

Proof. The result for finite $B$ follows from the proof of Theorem 2 which concerns unbounded capacity. Since the Markov process is finite and aperiodic there exists a stationary distribution. Moreover, the analysis for the unbounded case still holds for finite $B$ as long as $B \leq i^{*}+1$.

A new call will be rejected if all the $d$ choices select one of the edges with $\operatorname{load} i^{*}+1=\ln \ln n / \ln d+o(\ln \ln n / \ln d)$. With probability at least $1-n^{2}$, for each node, the number of incident edges with load at least $i^{*}+1$ is at 
most $2 \alpha_{i^{*}+1}$. Therefore the probability for a call to be rejected is no more than

$$
\frac{1}{n^{2}}+\left(\frac{2 \alpha_{i^{*}+1}}{n-1}+\frac{2 \alpha_{i^{*}+1}}{n-1}\right)^{d}=o\left(\frac{1}{n}\right)
$$

since $\alpha_{i^{*}+1}=10$.

\section{Lower Bound on the Performance of the DAR Algorithm}

To demonstrate the advantage of the balanced-allocation method we prove here a lower bound on the maximum channel load when requests are routed using the DAR algorithm. This bound shows an exponential gap between the capacity required by the balanced-allocation algorithm and the capacity required by the standard DAR algorithm for the same stream of inputs. Again we consider a complete network on $n$ nodes and $N=\left(\begin{array}{l}n \\ 2\end{array}\right)$ edges. Requests for connections between a given pair arrive according to a Poisson process with rate $\lambda$, the duration of a connection has an exponential distribution with expectation $1 / \mu$.

Theorem 6. Assume that all the edges have capacity $2 B$ circuits which can be a function of $n$. Then if we perform the DAR policy, edge capacity

$$
B=\Omega\left(\sqrt{\frac{\ln n}{d \ln \ln n}}\right), \quad \text { as } n \rightarrow \infty
$$

is necessary to ensure that a new call is not lost whp.

Proof. Recall that the edges have capacities $2 B$, capacity $B$ is used for direct connections, and the remaining capacity $B$ is used for alternative routes. We will compute a lower bound on the probability $P=P(B)$, that a request arriving at an arbitrary time $t$ is rejected.

We consider first the probability $P_{1}$ that the new call is not routed through the direct link. The process of routing calls through the direct link is similar to serving customers in an $M / M / B / B$ loss system (Poisson arrival, exponential service time, $B$ servers, up to $B$ customers in the system). Applying Erlang's loss formula (e.g., [9]),

$$
P_{1}=\frac{(\lambda / \mu)^{B}}{B !}\left(\sum_{i=0}^{B} \frac{(\lambda / \mu)^{B}}{i !}\right)^{-1} \geq e^{-\lambda / \mu} \frac{(\lambda / \mu)^{B}}{B !} .
$$


We will now estimate the probability $P_{2}$ that a request which was generated at time $t$ on edge $e$ and failed to use the direct link $e$, fails to be routed by an alternative path (i.e., all the $d$ attempts to find a non-saturated alternative path do not succeed). To give a lower bound to the failure probability, we consider a modified system that up to time $t$ behaves differently from the real one by rejecting more calls than the real one. Specifically, whenever the direct link is saturated it tries only one alternative path and if any of the edges of the path are saturated the call is rejected. Thus, clearly more calls are lost in the modified system and therefore fewer calls will exist at time $t$. Notice though that from time $t$ the system behaves in the regular way according to the DAR algorithm.

In order to estimate the probability $P_{2}$, we will try to lower bound the probability that at time $t$ all the $d$ alternative paths selected as candidates to serve the request have a saturated (as far as the bandwidth for alternative routes is concerned) edge, which is lower bounded by the probability that all the $d$ edges selected have the corresponding edge $e_{i}$ saturated (see Figure 1). For this we consider the system at some prior time $t-\tau$, for some $\tau$ that we will fix later. If some edge $e_{i}$ at that time point is saturated with $B$ calls, then the probability that all these calls remain in $e_{i}$ until time $t$ equals

$$
P_{\text {remain }}=e^{-\mu B \tau} \text {. }
$$

Assume now that edges $e_{i}$ and $e_{j}$ are not saturated at time $t-\tau$, and let $e_{i j}$ be the edge that joins them. We will try to compute the probability $P_{\text {new }}$ that a request is generated during $\tau$ by $e_{i j}$, routed through the alternative path $e_{i}-e_{j}$, and remained until time $t$. To simplify the argument, we ignore any already existing calls from $e_{i j}$ routed through that path-we are allowed to do that as these calls only increase the usage of $e_{i}$ and $e_{j}$.

We notice the following facts:

1. All the direct connections of $e_{i j}$ are occupied at time $t-\tau$ with probability $P_{1}$.

2. The time of a new event from edge $e_{i j}$ (either a new call or a termination of an existing call) is exponentially distributed with parameter $\lambda+B \mu$. Hence the probability of the first new event taking place in the period $\tau$ equals

$$
1-e^{-(\lambda+B \mu) \tau}
$$

3. Conditioning that there is such a new event, the probability that it is a new call (which will have to use an alternative path if all the direct 


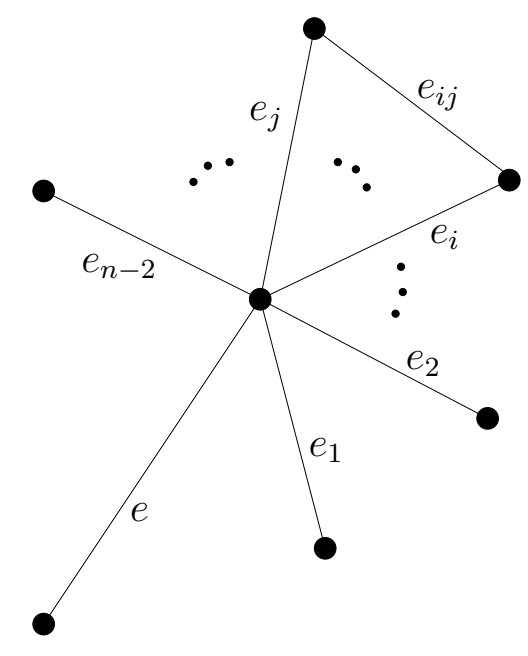

Figure 1: Edge $e$ with the new request and edges of the alternative paths.

links are occupied) is

$$
\frac{\lambda}{\lambda+B \mu}
$$

4. The probability that this call is served by the path $e_{i}-e_{j}$ is $1 /(n-2)$.

5. The probability that the call remains in the system until time $t$ is at least $e^{-\mu \tau}$.

Taking all these facts into account, we deduce that the probability that at time $t$ we have a call from the edge $e_{i j}$ in $e_{i}$ and $e_{j}$ is at least

$$
\begin{aligned}
P_{\text {new }} & =P_{1} \cdot\left(1-e^{-(\lambda+B \mu) \tau}\right) \cdot \frac{\lambda}{\lambda+B \mu} \cdot \frac{1}{n-2} \cdot e^{-\mu \tau} \\
& \geq e^{-\lambda / \mu} \frac{(\lambda / \mu)^{B}}{B !} \cdot\left(1-\frac{1}{e}\right) \cdot \frac{\lambda}{\lambda+B \mu} \cdot \frac{1}{n} \cdot e^{-\mu},
\end{aligned}
$$

where we have selected $\tau=1$ and hence $e^{-(\lambda+B \mu) \tau}<1 / e$ for large enough $n$.

For each edge $e_{i}$ there are $n-3$ potential sources $e_{i j}$ that are mutually independent. Notice however that if an edge $e_{k}$ is saturated then a diverted call from edge $e_{i k}$ that selected the path $e_{i}-e_{k}$ will be rejected and not contribute to the increase of the load of $e_{i}$. We perform the above counting as long as there are at least $n / 2-1$ non-saturated edges $e_{j}$. Then the 
probability that an edge $e_{i}$ is saturated at time $t$ is the minimum of $P_{\text {remain }}$ and

$$
P_{\text {full }}=\left(\begin{array}{c}
n / 2-1 \\
B
\end{array}\right) P_{\text {new }}^{B}\left(1-P_{\text {new }}\right)^{n / 2-B},
$$

and that minimum is always equal to $P_{\text {full }}$. Notice that a trivial upper bound for $P_{\text {new }}$ is $1 / n$ so

$$
P_{\text {full }} \geq\left(\begin{array}{c}
n / 2-1 \\
B
\end{array}\right) P_{\text {new }}^{B}\left(1-\frac{1}{n}\right)^{n / 2-B} .
$$

Let us now compute the probability $P_{2}$. There are at least $n / 2-1$ edges $e_{i}$ whose probability of being saturated is at least $P_{\text {full }}$, hence the probability that all the $d$ alternative paths selected contain one of the saturated edges $e_{i}$ is lower bounded by

$$
P_{2}=\left(\frac{n / 2-1}{n-2}\left(\begin{array}{c}
n / 2-d B-1 \\
B
\end{array}\right) P_{\text {new }}^{B}\left(1-\frac{1}{n}\right)^{n / 2-B}\right)^{d},
$$

where the extra term $-d B$ is needed to avoid dependences between the different edges $e_{i}$. Substituting the value for $P_{\text {new }}$ we get

$$
\begin{aligned}
P_{2} \geq & \frac{1}{2^{d}}\left(\begin{array}{c}
n / 2-d B-1 \\
B
\end{array}\right)^{d} e^{-d B \lambda / \mu} \cdot \frac{(\lambda / \mu)^{d B^{2}}}{(B !)^{d B}} \cdot\left(1-\frac{1}{e}\right)^{d B} . \\
& \left(\frac{\lambda}{\lambda+B \mu}\right)^{d B} \cdot \frac{1}{n^{d B}} \cdot e^{-d B \mu} \cdot\left(1-\frac{1}{n}\right)^{d n}
\end{aligned}
$$

Therefore the probability that the call generated at time $t$ is rejected is at least

$$
\begin{aligned}
P_{1} \cdot P_{2} \geq & e^{-\lambda / \mu} \frac{(\lambda / \mu)^{B}}{B !} \cdot \frac{1}{2^{d}}\left(\frac{n / 2-d B-1}{B}\right)^{d B} e^{-d B \lambda / \mu} \cdot \frac{(\lambda / \mu)^{d B^{2}}}{(B !)^{d B}} . \\
& \left(1-\frac{1}{e}\right)^{d B} \cdot\left(\frac{\lambda}{\lambda+B \mu}\right)^{d B} \cdot \frac{1}{n^{d B}} \cdot e^{-d B \mu} \cdot\left(\frac{1}{3^{d}}\right) \\
= & e^{-O\left(d B^{2} \ln B-d B^{2} \ln (\lambda / \mu)\right)}
\end{aligned}
$$

Therefore, in order to guarantee that a new call is not lost whp., the bandwidth must be at least

$$
B=\Omega\left(\sqrt{\frac{\ln n}{d \ln \ln n}}\right) .
$$




\section{References}

[1] G. R. Ash, R. H. Cardwell, and R. P. Murray. Design and optimization of networks with dynamic routing. BSTJ, 60, 8(8):1787-1820, 1981.

[2] Y. Azar, A. Broder, A. Karlin, and E. Upfal. Balanced allocations. In Proceedings of the 26th ACM Symposium on the Theory of Computing, pages 593-602, 1994.

[3] Y. Azar, A. Z. Broder, A. R. Karlin, and E. Upfal. Balanced allocations. SIAM Journal on Computing, 29(1):180-200, Feb. 2000.

[4] A. Z. Broder, A. Frieze, C. Lund, S. Phillips, and N. Reingold. Balanced allocations for tree-like inputs. Information Processing Letters, 55(6):329-332, Sept. 1995.

[5] D. Down, S. P. Meyn, and R. Tweedie. Exponential and uniform ergodicity of Markov processes. Ann. Probab., 23(4):1671-1691, 1996.

[6] R. J. Gibbens, P. J. Hunt, and F. P. Kelly. Bistability in communication networks. In G. R. Grimmet and D. J. A. Welsh, editors, Disorder in Physical Systems, pages 113-128. Oxford Univ. Press, New York, 1990.

[7] R. J. Gibbens, F. P. Kelly, and P. B. Key. Dynamic alternative routing. In M. E. Steenstrup, editor, Routing in Communications Networks, pages 13-47. Prentice Hall, 1995.

[8] P. J. Hunt and C. N. Laws. Asymptotically optimal loss network control. Mathematics of Operations Research, 18(4):880-900, 1993.

[9] F. P. Kelly. Loss networks. Annals of Applied Probability, 1(3):319-378, 1991.

[10] M. J. Luczak. Probability, Algorithms and Telecommunication Systems. DPhil thesis, Oxford University, 2000.

[11] M. J. Luczak, C. McDiarmid, and E. Upfal. On-line routing of random calls in networks. Probability Theory and Related Fields, 2002. To appear.

[12] M. J. Luczak and E. Upfal. Reducing network congestion and blocking probability through balanced allocation. In IEEE Symposium on Foundations of Computer Science, pages 587-595, 1999. 
[13] J. Martin and Y. Suhov. Fast Jackson networks. Ann. Appl. Probab., 9(3):854-870, 1999.

[14] S. P. Meyn and R. Tweedie. Stability of Markovian processes III: FosterLyapunov criteria for continuous-time processes. Adv. Appl. Probab., 25:518-548, 1993.

[15] S. P. Meyn and R. Tweedie. A survey of Foster-Lyapunov techniques for general state space Markov processes. In Proceedings of the Workshop on Stochastic Stability and Stochastic Stabilization, Metz, France, June 1993. Springer-Verlag, 1994.

[16] S. P. Meyn and R. L. Tweedie. Markov Chains and Stochastic Stability. Communications and Control Engineering Series. Springer-Verlag, London, New York, 1993.

[17] M. Mitzenmacher. The Power of Two Choices in Randomized Load Balancing. PhD thesis, University of California, Berkeley, August 1996.

[18] M. Mitzenmacher. On the analysis of randomized load balancing schemes. In Proceedings of the 9th Annual ACM Symposium on Parallel Algorithms and Architectures, pages 292-301, Newport, Rhode Island, June 22-25, 1997. SIGACT/SIGARCH and EATCS. Extended abstract.

[19] S. M. Ross. Applied Probability Models with Optimization Applications. Dover Publications, Reprint, 1970.

[20] S. M. Ross. A First Course in Probability. Macmillan, London, 5th edition, 1998.

[21] Y. Suhov and N. Vvedenskaya. Fast Jackson networks with dynamic routing. Problems of Information Transmission, 38(2):136-153, 2002.

[22] N. Vvedenskaya, R. Dobrushin, and F. Karpelevich. A queueing system with a choice of the shorter of two queues - an asymptotic approach. Problemy Peredachi Informatsii, 32(1):20-34, 1996. 